\title{
P115 Does Post-stroke White Coat Hypertension/Effect (WCH/E) Require Intensive Blood Pressure Management?
}

\author{
Fran Kirkham ${ }^{1 *}$, GN Nuredini ${ }^{2}$, A Saunders ${ }^{2}$, Erin Drazich ${ }^{1}$, Eva Bunting ${ }^{1}$, Philip Rankin ${ }^{1}, \mathrm{~K}$ Ali $^{1,2}$, M Okorie $^{1,2}$, \\ Chakravarthi Rajkumar ${ }^{1,2}$
}

${ }^{1}$ Department of Elderly Care and Stroke Medicine, Brighton and Sussex University Hospitals Trust, Brighton, UK

${ }^{2}$ Department of Medicine, Brighton and Sussex Medical School, Brighton, UK

\section{ABSTRACT}

Objective: International guidelines advocate conservative management of post-stroke white coat hypertension. The aims of this study were to investigate; i) does WCH/E result in increased stroke risk? and ii) is WCH/E associated with surrogate markers of cardiovascular risk?

Methods: The Arterial Stiffness In Lacunar Stroke and TIA (ASIST) study recruited 96 patients, aged over 40 years old, with a confirmed diagnosis of transient ischaemic attack (TIA) or lacunar stroke in the preceding 14 days. Patients were grouped by BP phenotypes. Thirty-four patients were excluded ( $n=6$ declined ABPM, $n=3$ masked hypertension, $n=25$ sustained hypertension). Thirty-two patients with normal BP (clinic BP $<140 / 90 \mathrm{mmHg}$ and day-time ABPM $<135 / 85 \mathrm{mmHg}$ ), and 30 patients with WCH/E (clinic BP $>140 / 90 \mathrm{mmHg}$ and day-time $\mathrm{ABPM}<135 / 85 \mathrm{mmHg}$ ) were recruited. Other surrogate markers measured were; Central aortic BP (SphygoCor, AtCor Medical), $\mathrm{QKD}_{100-60}$ interval and nocturnal dipping status (Diasys Integra II, Novocor).

Results: Compared to the normotensive cohort, patients with WCH/E were older, had a higher body mass index (BMI) and a larger proportion of patients were on anti-hypertensive medication. Both central systolic $(145 \pm 13 \mathrm{vs} 118 \pm 8, p<0.001)$ and diastolic $\mathrm{BP}(82 \pm 8$ vs $76 \pm 7, p=0.004)$ were higher in WCH/E. The WCH/E cohort also had more lacunar strokes $(p=0.039)$ (Table 1$)$.

Conclusion: In this population of post-stroke patients, $\mathrm{WCH} / \mathrm{E}$ was associated with higher prevalence of lacunar stroke. These individuals also had higher central pressures despite more patients being on anti-hypertensive treatment, suggesting that poststroke $\mathrm{WCH} / \mathrm{E}$ should be managed more aggressively.

Table 1

\begin{tabular}{|c|c|c|c|}
\hline & Normotension $(N=32)$ & $\mathrm{WCH} / \mathrm{E}(N=30)$ & Significance \\
\hline Male, $n(\%)$ & $21(66)$ & $22(73)$ & 0.511 \\
\hline Age (years) & $69.9 \pm 11.5$ & $75.7 \pm 93$ & 0.033 \\
\hline BMI $\left(\mathrm{kg} / \mathrm{m}^{2}\right)$ & $25 \pm 4$ & $28 \pm 4$ & 0.014 \\
\hline Anti-hypertensive use, $n$ (\%) & $19(59)$ & $23(77)$ & 0.146 \\
\hline Clinic SBP (mmHg) & $125 \pm 9$ & $155 \pm 13$ & $<0.001$ \\
\hline Clinic DBP (mmHg) & $75 \pm 7$ & $81 \pm 8$ & 0.003 \\
\hline Daytime systolic ABPM (mmHg) & $114 \pm 10$ & $121 \pm 10$ & 0.007 \\
\hline Daytime diastolic ABPM (mmHg) & $73 \pm 7$ & $72 \pm 7$ & 0.586 \\
\hline Central SBP (mmHg) & $118 \pm 8$ & $145 \pm 13$ & $<0.001$ \\
\hline Central DBP (mmHg) & $76 \pm 7$ & $82 \pm 8$ & 0.004 \\
\hline $\mathrm{QKD}_{100-60}$ interval (msec) & $208 \pm 18$ & $197 \pm 26$ & 0.114 \\
\hline Non-dipper, $n(\%)$ & $16(57)$ & $14(50)$ & 0.592 \\
\hline \multicolumn{4}{|l|}{ Stroke type } \\
\hline TIA, $n(\%)$ & $25(78)$ & $16(53)$ & \multirow{2}{*}{0.039} \\
\hline Lacunar, $n(\%)$ & $7(22)$ & $14(47)$ & \\
\hline
\end{tabular}

Data expressed as mean \pm standard deviation or number (percentage). Significance determined by $t$-test. Chi-squared used for: anti-hypertensive use, male gender, dipping status and stroke type.

(c) 2019 Association for Research into Arterial Structure and Physiology. Publishing services by Atlantis Press International B.V. This is an open access article distributed under the CC BY-NC 4.0 license (http://creativecommons.org/licenses/by-nc/4.0/). 